\title{
Les cartes d'extension PCBmod : conception et applications pédagogiques VHDL et micro-assemblage
}

\author{
A. Guédon-Gracia, H. Debéda, J. Tomas \\ IMS et pôle CNFM de Bordeaux (PCB), Université de Bordeaux, Bordeaux INP \\ Contact : jean.tomas@ims-bordeaux.fr
}

\begin{abstract}
Nous avons développé et fabriqué des cartes d'extension compatibles avec les connecteurs Pmod des cartes de développement FPGA de la société Digilent. Ces cartes sont appelées PCBmod et sont au nombre de quatre actuellement. Elles intègrent respectivement des convertisseurs, des capteurs de température et de pression ainsi qu'un circuit date-calendrier; tous ces composants possèdent un protocole de communication SPI. Ces PCBmod sont utilisés au niveau Master en tant qu'exemple d'application lors du stage micro-assemblage, et en tant que périphériques divers et variés lors de TP et projet VHDL.
\end{abstract}

Mots clés : micro-assemblage, brasage, CMS, VHDL, FPGA, Pmod, SPI

\section{Introduction}

Traditionnellement, le pôle CNFM de Bordeaux (PCB) propose aux étudiants des filières électroniques un stage de micro-assemblage (1). De plus, les étudiants niveau Master effectuent des projets VHDL, dont le sujet porte majoritairement sur le développement de l'interface de communication entre le FPGA (Field-Programmable Gate Array) et le circuit qui se trouve sur une carte d'extension. Le pôle PCB dispose de cartes de développement FPGA de la société Digilent (Nexys3 et Basys3), acquises avantageusement grâce à l'opération « une carte - un étudiant » sponsorisée par la société Xilinx et le GIP-CNFM. Les cartes d'extension utilisées sont des cartes de type Pmod (2), également développées par la société Digilent. L'idée, qui s'est naturellement imposée, est d'associer les deux activités précédentes et donc, de proposer le micro-assemblage d'une carte d'extension et de la tester fonctionnellement en utilisant le pilote VHDL préalablement développé et validé.

De nouvelles cartes, compatibles Pmod, ont été conçues avec le soutien du GIPCNFM (3) et de l'ANR IDEFI-FINMINA (4). Nous les appelons PCBmod. Ces cartes sont au nombre de quatre actuellement. La première est dédiée à la conversion numérique-analogique, les trois autres intègrent des composants à protocole Serial Peripheral Interface (SPI), respectivement un capteur de température, un capteur de pression et une horloge-calendrier.

Cet article présente au paragraphe II le détail de chacune des cartes. Puis, suit le descriptif des applications pédagogiques, à savoir les TP/projets VHDL basés sur le développement de l'interface de communication SPI et le déroulement du stage microassemblage, avant de conclure.

La première promotion qui effectuera le stage micro-assemblage est le Master 2 « Ingénierie des systèmes embarqués » de l'Université de Bordeaux. D'autres spécialités ainsi que des étudiants niveau Master 1 pourront suivre ces travaux pratiques. 


\section{Les cartes d'extension PCBmod}

Dans ce paragraphe, nous détaillons les quatre cartes conçues :

- PCBmodANNA: conversion Analogique-Numérique et NumériqueAnalogique ;

- PCBmodRTCC: horloge temps réel et calendrier (Real Time Clock \& Calendar);

- PCBmodBARO : capteur de pression ;

- PCBmodTEMP : capteur de température.

Les communications séries de tous ces modules se font par un protocole SPI. À remarquer que seuls des Pmod avec protocole I2C existent chez le fournisseur Digilent pour les trois derniers modules. Ces cartes ont été créées en respectant le format Pmod présent sur les cartes Nexys3 et Basys3 (5). Elles sont double couches, alimentées par la carte FPGA sous 3,3Volt et possèdent un connecteur vertical permettant la connexion d'un analyseur logique afin de visualiser la communication, SPI pour l'heure.

\section{PCBmodANNA}

La carte PCBmodANNA vient en remplacement d'une carte similaire qui était destinée aux cartes de développement «Spartan3E starter kit». Elle est uniquement dédiée à l'étude de la communication SPI et ne fait pas l'objet du stage micro-assemblage.

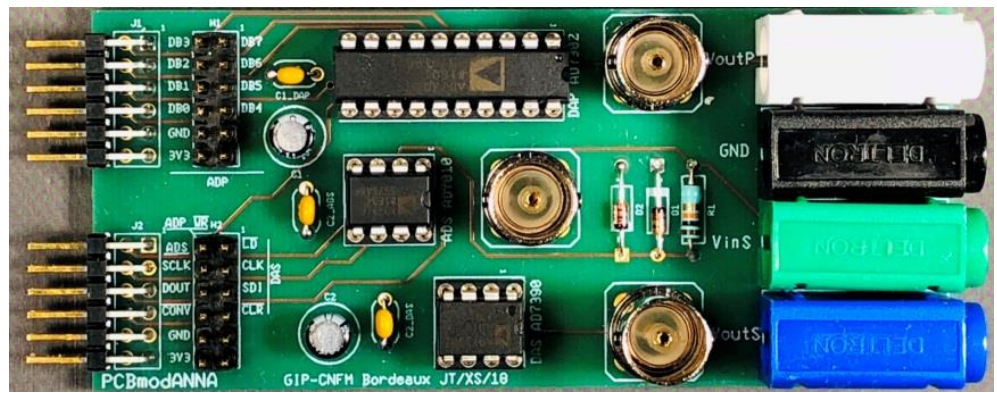

Fig.1. Photographie de la carte PCBmodANNA, dimension 98mm x 43mm.

Elle comprend :

- Un convertisseur numérique analogique parallèle 8 bits AD7302 ;

- Un convertisseur analogique numérique série 10 bits AD7810 ;

- Un convertisseur numérique analogique série 12 bits AD7390.

La carte dispose de deux connecteurs coudés type Pmod J1 et J2, de deux connecteurs verticaux $\mathrm{H} 1$ et $\mathrm{H} 2$, des sorties de type BNC ou de type banane. Enfin le convertisseur analogique numérique possède un circuit écrêteur sur son entrée analogique, par contre il n'y pas de filtre anti-repliement.

La figure 2 illustre les deux faces du circuit imprimé conçu pour la carte PCBmodANNA sous le logiciel UltiBoard de National Instrument. 

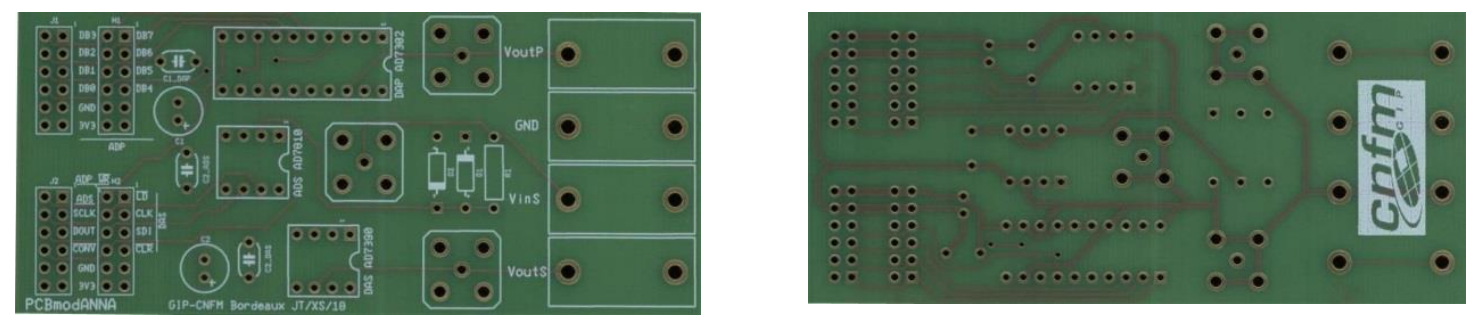

Fig.2. Circuit imprimé, recto et verso, de la carte PCBmodANNA, dimensions 98mm x 43mm.

\section{PCBmodRTCC}

La carte PCBmodRTCC utilise le composant MCP79510 qui comprend une horloge, un calendrier et une gestion d'alarme. Une pile bouton permet la sauvegarde des réglages. Le schéma électrique est donné en figure 3.

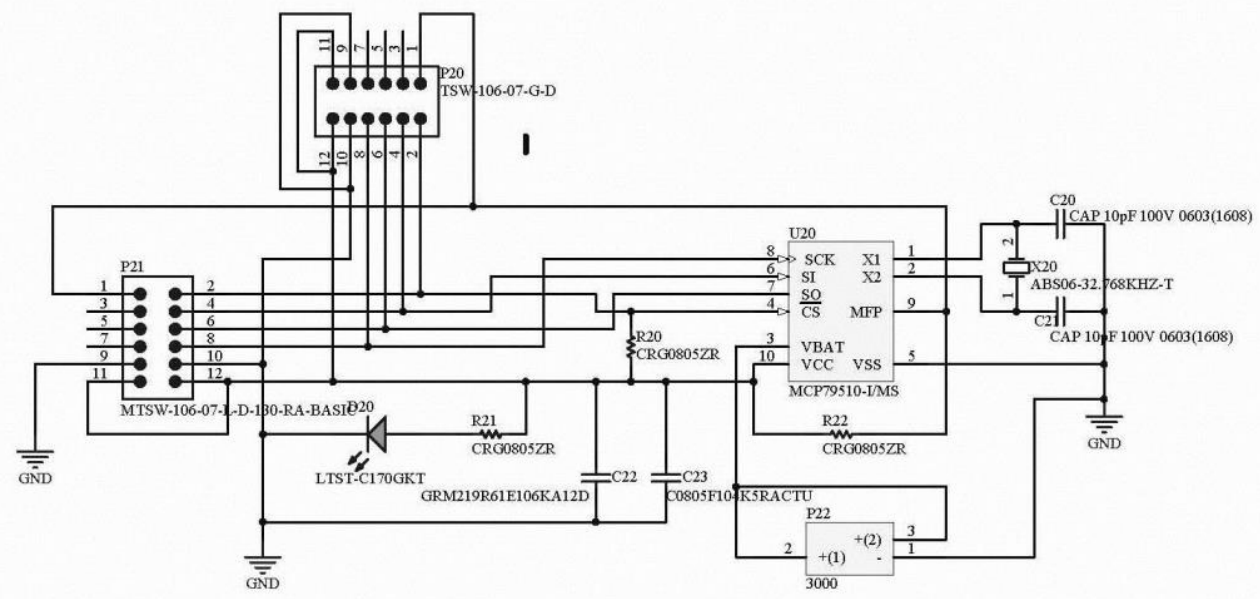

Fig.3. Schéma électrique de la carte PCBmodRTCC.

La figure 4 illustre les deux faces du circuit imprimé conçu pour la carte PCBmodRTCC.
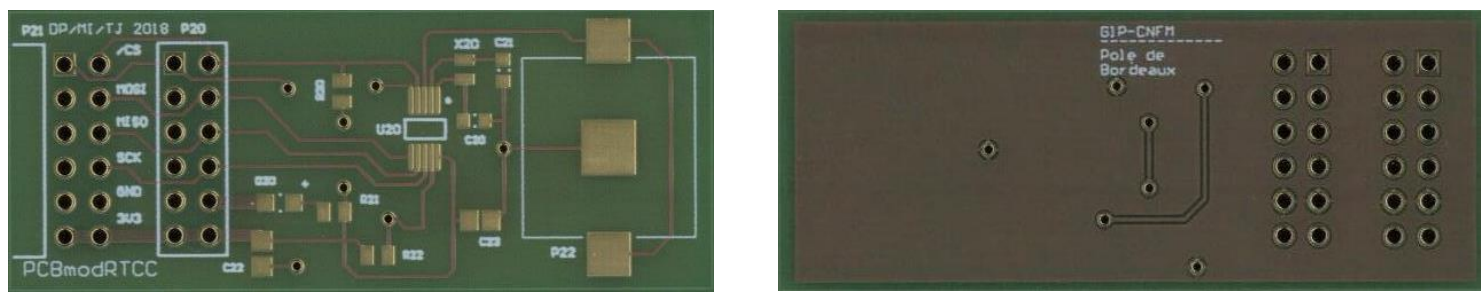

Fig.4. Circuit imprimé, recto et verso, de la carte PCBmodRTCC, dimension 52mm x 20mm.

Cette carte de dimensions $2 \mathrm{~mm}$ x $20 \mathrm{~mm}$ x $1,6 \mathrm{~mm}$, double face avec plan de masse, a été fabriquée par la société PCB-Pool. Elle a été livrée avec un masque de sérigraphie qui doit servir au dépôt automatique de la crème à braser. Les 2 faces sont recouvertes d'un vernis épargne vert photosensible. La finition choisie des plots de connexion, sur lesquels sont brasés les CMS (Composants Montés en Surface), est de type ENIG (nickel avec un flash d'or). Cette finition permet d'assurer une meilleure connexion électro-mécanique et électrique. Le flash d'or assure en effet une meilleure mouillabilité de la brasure et le nickel joue le rôle de barrière de diffusion, ce qui améliore la fiabilité du circuit dans le 
temps. Des trous métallisés de 40 mil de diamètre ont été créés pour accueillir les 2 connecteurs qui seront insérés. Les caractéristiques principales des composants CMS choisis pour la réalisation de carte sont donnés tableau I. Pour l'étape de brasage, les tailles millimétriques de ces composants permettent leur report manuel sur la carte (à l'aide de précelles). Ceci est donc adapté au type de TP proposé, puisque les étudiants doivent manipuler et observer les différents boitiers CMS.

TABLEAU I. Caractéristiques des composants utilisés pour la carte PCmodRTCC.

\begin{tabular}{|c|c|c|c|}
\hline \multicolumn{2}{|c|}{ Composants } & Nomencl./valeur & Boitier \\
\hline \multirow{7}{*}{ Dipôles } & \multirow{3}{*}{ Résistances } & $\mathbf{R 2 0} / 10 \mathrm{k} \Omega$ & \multirow{3}{*}{$\begin{array}{l}\text { Chip CMS 0805/ } \\
\text { 2,0mmx1,2mm }\end{array}$} \\
\hline & & $\mathbf{R 2 1} / 10 \mathrm{k} \Omega$ & \\
\hline & & $\mathbf{R 2 2} / 10 \mathrm{k} \Omega$ & \\
\hline & \multirow{4}{*}{$\begin{array}{c}\text { Condensateurs } \\
\text { céramiques multicouche } \\
\text { MLCC }\end{array}$} & C20 / 10pF & Chip CMS 0805/ \\
\hline & & C21 / 10pF & $2,0 \mathrm{mmx} 1,2 \mathrm{~mm}$ \\
\hline & & $\mathbf{C 2 2} / 10 \mu \mathrm{F}$ & Chip 0805/ \\
\hline & & $\mathbf{C 2 3} / 100 \mathrm{nF}$ & $2,0 \mathrm{mmx} 1,2 \mathrm{~mm}$ \\
\hline LED & $\begin{array}{l}\text { Diode } \\
\text { électroluminescente }\end{array}$ & $\begin{array}{c}\text { D20 / } \\
\text { bleu, } 475 \mathrm{~nm}\end{array}$ & $\begin{array}{c}\text { Chip CMS 0805/ } \\
2 \mathrm{~mm} \times 1,2 \mathrm{~mm} \times 1,1 \mathrm{~mm}\end{array}$ \\
\hline \multirow{2}{*}{ Connecteurs } & $\begin{array}{l}\text { Embase à broches, } \\
\text { Molex }\end{array}$ & P20 / 3A max & $\begin{array}{l}12 \text { Contacts, } 2 \text { Rangées, entraxe } \\
2,54 \mathrm{~mm} \text {, vertical }\end{array}$ \\
\hline & $\begin{array}{c}\text { Embase à broches, } \\
\text { Molex }\end{array}$ & P21 / 3A max & $\begin{array}{c}12 \text { Contacts, } 2 \text { Rangées, entraxe } \\
2,54 \mathrm{~mm} \text {, angle droit } \\
\end{array}$ \\
\hline \multirow{2}{*}{ Circuits } & $\begin{array}{l}\text { Horloge en temps réel, } \\
\text { Calendrier }\end{array}$ & U20 / MCP79510 & MSOP 10 broches \\
\hline & Quartz, Micro Crystal &  & CMS $2 \times 1,2 \times 0,6 \mathrm{~mm}$ \\
\hline Support pile & $\begin{array}{l}\text { Contact pour pile } \\
\text { CR1216 }\end{array}$ & $\mathbf{P 2 2}$ & $\begin{array}{l}\text { Boitier CMS métallique nickel-étain/ } \\
18,9 \mathrm{~mm} \times 12,1 \mathrm{~mm} \times 3,2 \mathrm{~mm}\end{array}$ \\
\hline $\begin{array}{c}\text { Carte } \\
\text { PCBmodRTCC }\end{array}$ & \multicolumn{2}{|c|}{ Circuit imprimé 2 faces } & $\begin{array}{c}\text { Vernis épargne + finition ENIG / } \\
52 \mathrm{~mm} \times 20 \mathrm{~mm} \times 1,6 \mathrm{~mm}\end{array}$ \\
\hline
\end{tabular}

Un stagiaire niveau Licence 1 SPI a effectué l'assemblage de la première carte (figure 5).

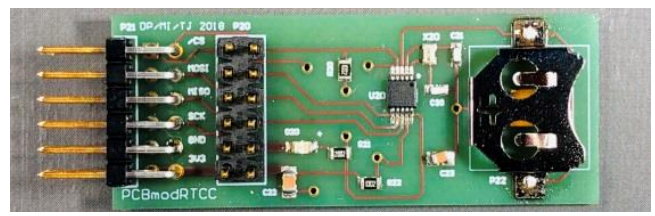

Fig.5. Carte PCBmodRTCC assemblée au sein du pôle PCB, dimension 52mm x 52mm x 1,6mm.

\section{PCBmodBARO}

La carte PCBmodBARO est construite autour du composant MPL115A1 qui est un capteur de pression allant de 50 à $115 \mathrm{kPa}$ avec une précision de $1 \mathrm{kPa}$. Le schéma électrique ainsi que le dessin des masques ont été réalisés. Le circuit imprimé est en cours de fabrication.

\section{PCBmodTEMP}

La carte PCBmodTEMP est construite autour du composant TC72 qui est un capteur de température de plage $\left[-40^{\circ} \mathrm{C} ;-85^{\circ} \mathrm{C}\right]$ avec une résolution sur 10 bits. Le schéma électrique ainsi que le dessin des masques ont été réalisés. Le circuit imprimé est en cours de fabrication. 
Ces trois dernières cartes ont été développées sous Altium Designer de la société éponyme par un stagiaire du Master 1 Ingénierie des Systèmes Embarqués.

\section{III.Applications pédagogiques}

La carte PCBmodANNA est utilisée en TP VHDL. Les cartes PCBmodRTCC, PCBmodTEMP et PCBmodBARO sont destinées à être utilisées dans le cadre de projet VHDL et du stage de micro-assemblage.

VHDL : écriture de l'interface de communication SPI

En première année de Master, l'accent est mis sur la conception de systèmes numériques en utilisant les machines à états finis. La disponibilité de nombreux composants (capteurs, convertisseurs, ...) à liaison SPI permet de diversifier les exemples d'application et de personnaliser les projets. La méthodologie à suivre est la suivante :

- Spécification technique du besoin ;

- Analyse de la datasheet du capteur ou du convertisseur ;

- Symbole du composant «interface SPI»;

- Extraction du timing propre à la liaison SPI et adaptation aux contraintes de la carte FPGA de développement ;

- Conception de la machine à états finie ;

- Dessin de l'architecture du composant « interface SPI »;

- Codage VHDL ;

- Simulation et vérification du timing.

Deux séances de TP VHDL (durée unitaire de 2h50) sont consacrées à l'élaboration du composant SPI_AD7390 qui gère la liaison SPI entre le FPGA et le convertisseur numérique-analogique série de la carte PCBmodANNA ainsi qu'à la mise en œuvre sur la carte Nexys3 pour vérifier son bon fonctionnement.

La communication SPI est simple car seul le canal MOSI (Master Out Slave In) est utilisé ; les données sont uniquement envoyées du FPGA vers le convertisseur numérique analogique.

Un exemple de projet qui sera proposé est la construction d'une station météo. Ce projet est lui-même découpé en sous projets, chacun d'entre eux concernant l'acquisition de données de type heure, date, température et pression atmosphérique fournies respectivement par les cartes PCBmodRTCC, PCBmodTEMP et PCBmodBARO. Dans le cas du PCBmodRTCC, les deux canaux MOSI et MISO seront utilisés, le premier pour la mise à l'heure du composant et pour la configuration d'une éventuelle alarme, le second pour l'acquisition des données calendaires et horaires.

\section{$\underline{\text { Micro-assemblage : brasage des composants montés en surface }}$}

Les étapes suivantes vont permettre de décrire l'assemblage de la carte PCBmodRTCC. Les étudiants les réaliseront toutes au sein de la plateforme assemblage du laboratoire IMS sur une demi-journée.

- Etape 1 : dépôt manuel de la crème à braser $\mathrm{SnAgCu}(c f$. figure 6)

La composition de $\mathrm{SnAg} 3 \mathrm{Cu} 0,5$ ou appelé SAC 305 est 96,5\% d'étain, 3\% d'argent et $0,5 \%$ de cuivre. Cet alliage est un alliage eutectique qui passe directement de l'état solide à l'état liquide à $217^{\circ} \mathrm{C}$. Il sera déposé sur les plots de cuivre avec une aiguille sous la binoculaire. 
- Etape 2 : placement des composants montés en surface (CMS) et du contact de pile avec une précelle (fig.6)

La documentation sur les composants «non symétriques » tels que la diode D20 ou le boîtier U20 (voir tableau I) doit être consultée pour bien positionner les composants.

- Etape 3 : refusion de la crème à braser dans le four à phase vapeur (fig.6)

Le principe de ce four est qu'un liquide inerte (Galden®) est porté à ébullition et sa vapeur se dépose par condensation sur toute la carte. Ceci assure une bonne homogénéité du chauffage (diffusion de la vapeur partout) et un bon contrôle de la température. Ainsi le risque d'avoir des joints mal brasés ou des composants endommagés par une température mal contrôlée est nul.
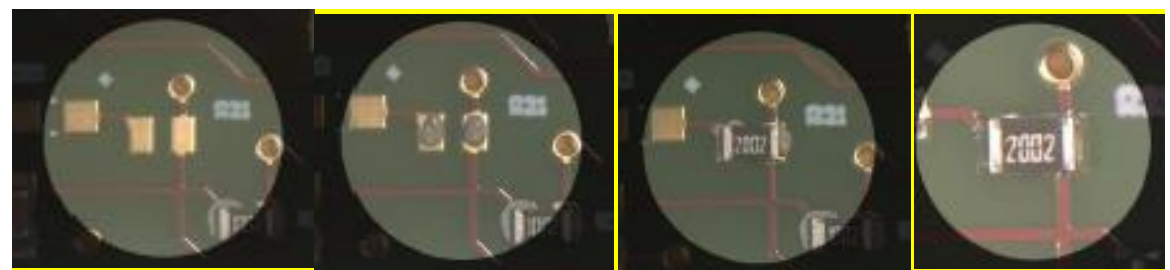

Fig.6. Différentes étapes d'assemblage : avant, après dépôt de la crème, après placement des composants et après refusion.

- Etape 4 : inspection visuelle des joints brasés sous binoculaire.

Il faut s'assurer de l'absence de courts-circuits et de la bonne connexion entre les composants et la carte.

- Etape 5 : positionnement des composants traversants PTH (Pin Through Hole)

Les PTH sont ici des embases à broches ou connecteurs. Ils sont assemblés sur la carte avec un fer à souder et un fil à base de $\mathrm{SnAgCu}$. Les brasures sont faites sur la face arrière de la carte.

\section{Conclusion}

Cet article présente le développement de cartes d'extension PCBmod qui servent de supports pédagogiques à la fois pour l'enseignement du VHDL niveau Master sous forme de TP et projets, ainsi que pour le stage de micro-assemblage en tant qu'exemple de réalisation.

\section{Remerciements}

Nous tenons à remercier M. A. Cheikh-Brahim (Licence 1 SPI - stage d'excellence) pour l'assemblage du premier prototype, M. P. Drouot (Master1 MA-ISE - stage) pour la conception des cartes. Les travaux ont bénéficié du soutien du GIP-CNFM et donc du Ministère de l'Enseignement Supérieur et de la Recherche, ainsi que du programme IDEFI-FINMINA.

\section{Références}

1. Debéda, H., Fadel, L., Favre, I., Lachaud, J.-L., and J. Tomas. Vers l'électronique imprimée à l'IMS Bordeaux - plateforme technologique TAMIS (Technologies Alternatives aux MIcrosystèmes Silicium). J3eA, 14 (2015)

2. https://store.digilentinc.com/pmod-expansion-modules/pmod-boards/.

3. GIP-CNFM: Groupement d'Intérêt Public - Coordination Nationale pour la formation en Microélectronique et en nanotechnologies. Website: http://wwww.cnfm.fr 
4. IDEFI-FINMINA : Initiative d'Excellence - Formation Innovante en MIcroélectronique et Nanotechnologies, ANR-11-IDFI-0017.

Website: http://www.cnfm.fr/VersionFrancaise/actualites/ FINMINA.htm

5. Société Digilent : https://reference.digilentinc.com/_media/pmod:pmod:originalPmodInterface Specification.pdf 